\title{
Overview of the anatomical basis of the piriformis syndrome-dissection with magnetic resonance correlation
}

\author{
OFELIA-COSTINA GOIDESCU ${ }^{1}$, MIHALY ENYEDI ${ }^{2,3}$, ADRIAN-DANIEL TULIN ${ }^{2}$, \\ RALUCA TULIN $^{2}$, ILEANA ADELA VACAROIU ${ }^{4}$, ADRIANA ELENA NICA ${ }^{5}$, DORIN DRAGOS ${ }^{6}$, \\ DORIN IONESCU $^{6}$, DRAGOS GEORGESCU ${ }^{7}$, ADRIAN MIRON $^{7}$ and FLORIN-MIHAIL FILIPOIU ${ }^{2}$ \\ ${ }^{1}$ Doctoral School, Faculty of Medicine, 'Carol Davila' University of Medicine and Pharmacy; \\ ${ }^{2}$ Department of Morphological Sciences, Discipline of Anatomy, Faculty of Medicine, \\ 'Carol Davila' University of Medicine and Pharmacy, 020021 Bucharest; ${ }^{3}$ 'Victor Babeş' Diagnosis and \\ Treatment Center, 030303 Bucharest; Departments of ${ }^{4}$ Nephrology and ${ }^{5}$ Orthopedics, Faculty of Medicine, \\ 'Carol Davila' University of Medicine and Pharmacy; ${ }^{6}$ Department of Medical Semiology, \\ Discipline of Internal Medicine I and Nephrology, ${ }^{7}$ Discipline of General Surgery, Faculty of Medicine, \\ 'Carol Davila' University of Medicine and Pharmacy, 020021 Bucharest, Romania
}

Received September 8, 2021; Accepted October 8, 2021

DOI: $10.3892 / \mathrm{etm} .2021 .11036$

\begin{abstract}
The piriformis syndrome is one of the most commonly misdiagnosed causes of lower back and gluteal pain caused by the compression of the sciatic nerve and the internal pudendal neurovascular bundle by the piriformis muscle. Although this syndrome was first suggested over 90 years ago, its diagnosis still represents a challenge for clinicians. In the present study, dissection was used to determine the intra- and extrapelvic anatomical course of the internal pudendal nerve and the data were compared with the information obtainable through MRI examination, in order to identify the piriformis syndrome and to differentiate it from other causes of internal pudendal neuralgia. Thorough dissections of the pelvis and deep gluteal region were conducted on female cadavers, which were correlated with MRI scans, in order to describe the course of the internal pudendal nerve in contact with the piriformis muscle. The dissection findings and MRI scans obtained allowed us to describe and demonstrate the compression points along the course of the sciatic nerve and the internal pudendal bundle, the anatomical correlations between the piriformis muscle and the nervous structures around it, emphasizing the areas most susceptible to possible nerve impingement syndromes. In the anatomic trajectory of the sciatic nerve and the internal pudendal bundle there are multiple contact points with anatomical structures that may lead to compression of the
\end{abstract}

Correspondence to: $\operatorname{Dr}$ Mihaly Enyedi, Department of Morphological Sciences, Discipline of Anatomy, Faculty of Medicine, 'Carol Davila' University of Medicine and Pharmacy, 8 Eroii Sanitari Bvd, 020021 Bucharest, Romania

E-mail: mihai_eny@yahoo.com

Key words: piriformis syndrome, deep gluteal syndrome, sciatic nerve, lower back pain, dissection, magnetic resonance imaging nerve structures, generating symptoms that comprise the piriformis syndrome. The present study sought to establish clear osseous landmarks that may help evaluate these associations and possible nerve compressions on pelvic MRI examination.

\section{Introduction}

The piriformis is, as the name suggests, a pear-shaped, flat muscle located in the deep gluteal region. Its origin is usually located on the anterior surface of the lateral processes of the second to fourth sacral segments (1), on the anterior part of the capsule of the sacroiliac joint, on the gluteal surface of the ilium in the vicinity of the greater sciatic notch and, occasionally, on the sacrotuberous ligament. The insertion is located on the medial surface of the superior aspect of the greater trochanter of the femur, where its tendon fuses with the tendons of the superior gemellus, obturator internus and inferior gemellus muscles to form the conjoint tendon. The piriformis muscle passes from the posterior pelvic region to the gluteal region through the greater sciatic notch. Its nerve supply passes through the anterior branches of the S2 and S3 sacral nerves arising from the sacral plexus $(2,3)$.

The sciatic nerve is the widest peripheral nerve of the body and the terminal branch of the sacral plexus. It usually runs as a single trunk through the pelvic cavity, the gluteal region and the posterior region of the thigh, until it reaches the superior angle of the popliteal fossa, where it divides into the tibial and the common peroneal nerves (1).

On occasion, the close anatomical relationship between the piriformis muscle and the sciatic nerve may lead to the compression of the latter, described as the piriformis syndrome (4).

There are wide variations in the relationship between the piriformis muscle and the sciatic nerve, with six possible anatomical associations (5-7): i) The sciatic nerve passes below the piriformis muscle; ii) the divided sciatic nerve passes 
through and below the muscle; iii) the divided sciatic nerve passes above and below the muscle; iv) the undivided sciatic nerve passes through the muscle; v) the divided sciatic nerve passes above and through the muscle; and vi) presence of a smaller accessory piriformis muscle with a separate tendon, located below them main piriformis muscle, with the sciatic nerve passing between the two. Among all these variants, the most common is the sciatic nerve passing undivided below the piriformis muscle, found in $84 \%$ of 120 cadavers dissected by Beaton and Anson (6) and in $78 \%$ of 130 cadavers dissected by Pećina (7), followed by the variant with the divided sciatic nerve passing through and below the piriformis muscle, found in $12 \%$ of the cases in the study of Beaton and Anson (6) and in $21 \%$ in the study of Pećina (7).

The piriformis syndrome is often misdiagnosed, being mainly confused with discogenic compression neuropathy or with other causes of gluteal or hip pain, including trochanteric bursitis, sacroileitis and sciatica (3).

Sciatica is defined as referred pain felt in the lower limb, throughout the distribution of the sciatic nerve. Low back pain is also a frequently reported symptom (4).

The treatment for this syndrome can be either non-surgical or surgical (8).

In 1928, Yeoman (9) was the first to describe the relationship between the piriformis muscle and the sciatic nerve as an etiological factor of low back pain and sciatic pain.

In 1934, Freiburg and Vinke (10) stated that inflammatory processes within the sacroiliac joint primarily affect the piriformis muscle and fascia, followed by irritation of the elements of the lumbar and sacral plexuses.

Four years later in 1938, Beaton and Anson (11) conducted a study based on cadaver dissections, concluding that the spasm of the piriformis muscle may be a cause of irritation of the sciatic nerve.

The term 'piriformis syndrome' was introduced in 1947 by Robinson (12), who used it to define the sciatic pain related to an abnormal piriformis muscle, usually of traumatic origin.

The piriformis syndrome has long been considered as a clinical diagnosis and, occasionally, a diagnosis of exclusion, for which no clear diagnostic criteria have been determined to date (12).

A recent clinical study by Hopayian and Danielyan (13) states that there are four symptoms that define the syndrome: Gluteal pain, pain exacerbation upon sitting, external tenderness near the greater sciatic notch and limitation of straight leg raising due to pain.

The deep gluteal syndrome is a condition characterized by a set of clinical symptoms and other semiotic data occurring in isolation and in combination (14-16), with the most common being posterior pain, tenderness and/or dysesthesia in the gluteal region, hip area or posterior thigh region. The symptoms are usually unilateral, but they can occur bilaterally. Other symptoms reported by patients include intolerance to sitting for $>20-30 \mathrm{~min}$, limping, loss of sensitivity in the affected limb, and pain at night that improves during the day (15-17).

The piriformis syndrome is considered as a subgroup of the deep gluteal syndrome (15), which occurs due to several conditions: Asymmetrical hypertrophy of the piriformis muscle with anterior displacement of the sciatic nerve; dynamic sciatic nerve entrapment by the piriformis muscle; and variants in the course of the sciatic nerve related to the piriformis muscle.

In addition to the piriformis syndrome, the deep gluteal syndrome also includes the gemelli-obturator internus syndrome, the ischiofemoral impingement syndrome and the proximal hamstring syndrome (18).

The aim of the present study was to combine cadaveric dissections and pelvic MRI examinations to establish clear osseous landmarks that may help evaluate the anatomical associations between the sciatic nerve and the piriformis muscle and identify possible nerve compression points.

\section{Materials and methods}

Study intent. The controversies regarding the existence of the piriformis syndrome, the high rate of misdiagnosed and mistreated cases and the complex anatomy of the pelvic and gluteal regions require further morphological studies. This led to the concept of conducting combined anatomical and imaging studies, using dissection of the region and comparison to the images obtained through MRI examination, in the hope that the anatomical study should help physicians with clinical and imaging diagnosis.

The study was intended to combine direct anatomical observation and an analysis of MRI scans in order to explain the anatomical background of the development of the piriformis syndrome.

Cadaveric dissection. For the purposes of the present study, five female cadavers obtained from the Anatomy Department of the 'Carol Davila' University of Medicine and Pharmacy (Bucharest, Romania) were dissected in an appropriate manner so as to emphasize the morphology and the anatomical relations of the piriformis muscle, the sciatic nerve and the internal pudendal neurovascular bundle.

In order to obtain a favorable approach, the pelvis of the cadavers was separated from the rest of the body by using transverse cross sections at the following levels: Cranially through the L4-L5 intervertebral disc and caudally at $10 \mathrm{~cm}$ below the greater trochanter. The obtained anatomical specimen was then sagittally sectioned $1-2 \mathrm{~cm}$ lateral to the pubic symphysis.

The dissection was conducted in anatomical planes all the way to the level of the levator ani muscle. This muscle was disinserted from the fascia of the internal obturator muscle and reflected medially in order to create good access towards the ischiorectal fossa. The sacral origins of the piriformis muscle and the trunks of origin of the sciatic nerve were identified and separated through careful dissection.

In another plane, the origins and the organization pattern of the pudendal nerve, the sacrospinous ligament, the coccygeus muscle and the perineal course of the internal pudendal neurovascular bundle were dissected and separated from the surrounding anatomical elements.

The superior and inferior gluteal artery and the internal pudendal artery, which are branches of the internal iliac artery, were preserved and highlighted.

The internal obturator muscle was been carefully dissected, emphasizing the orientation of its origins and the manner in which its fascicles unite to exit the pelvis below the ischial spine. The infrapiriform space was also dissected to 


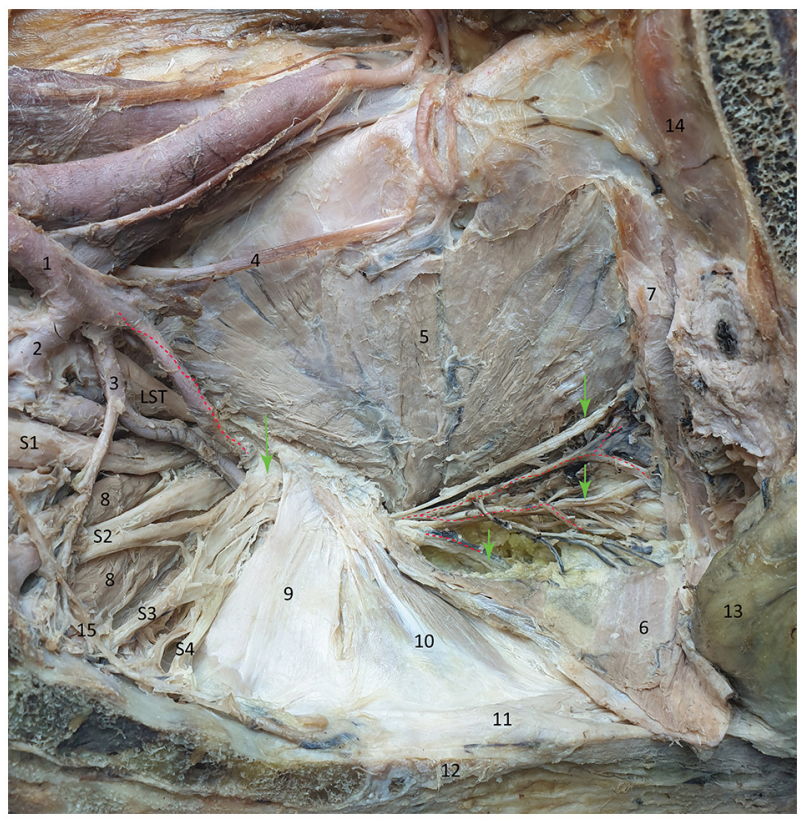

Figure 1. Internal view of the pelvic wall. The red dotted line marks the internal pudendal artery and its branches; the green arrows mark the internal pudendal nerve and its branches: Dorsal nerve of clitoris, perineal, inferior rectal nerves; S1-S4, anterior branches of the sacral plexus. 1, Internal iliac artery; 2, superior gluteal artery; 3 , inferior gluteal artery; 4 , obturator nerve; 5 , internal obturator muscle; 6 , levator ani muscle (reflected); 7 , levator ani muscle (cut); 8 , piriformis muscle; 9 , sacrospinous ligament; 10, coccygeus muscle; 11, anococcygeal ligament; 12 , coccyx bone 13 , rectum. 14, pubic bone; 15 , sympathetic trunk.

emphasize the anatomical relations of the sciatic and internal pudendal nerves.

In order to demonstrate the perineal course of the internal pudendal nerve, dissections of the gluteal region were conducted through a posterior approach and the nerve was highlighted following removal of the gluteus maximus muscle.

MRI scans. The second part of the study included the analysis of 10 MRI scans selected from the patients of the 'Victor Babeş' Diagnosis and Treatment Center, the clinic where one of the authors works in. The selection criteria comprised the absence of any pathological findings in the pelvic and gluteal areas and the high quality of the images obtained during the examination. This allowed us to perform a thorough analysis and correctly evaluate the association with the aspects emphasized through dissection. The landmarks useful for identifying the critical relations of the nervous structures were identified based on data acquired through dissection.

The MRI equipment utilized for obtaining the images included in the present study was a Siemens Magnetom Avanto 1.5T (Siemens Healthineers). The Body and the Flex Large coils were used. The examination protocol included the following sequences: Axial T2 turbo spin echo (TSE), axial T1 SE, coronal short tau inversion recovery (STIR), coronal T1 TSE and sagittal T2 TSE.

\section{Results}

Observations during dissection. For the purposes of the first part of this study, all the dissection specimens that were

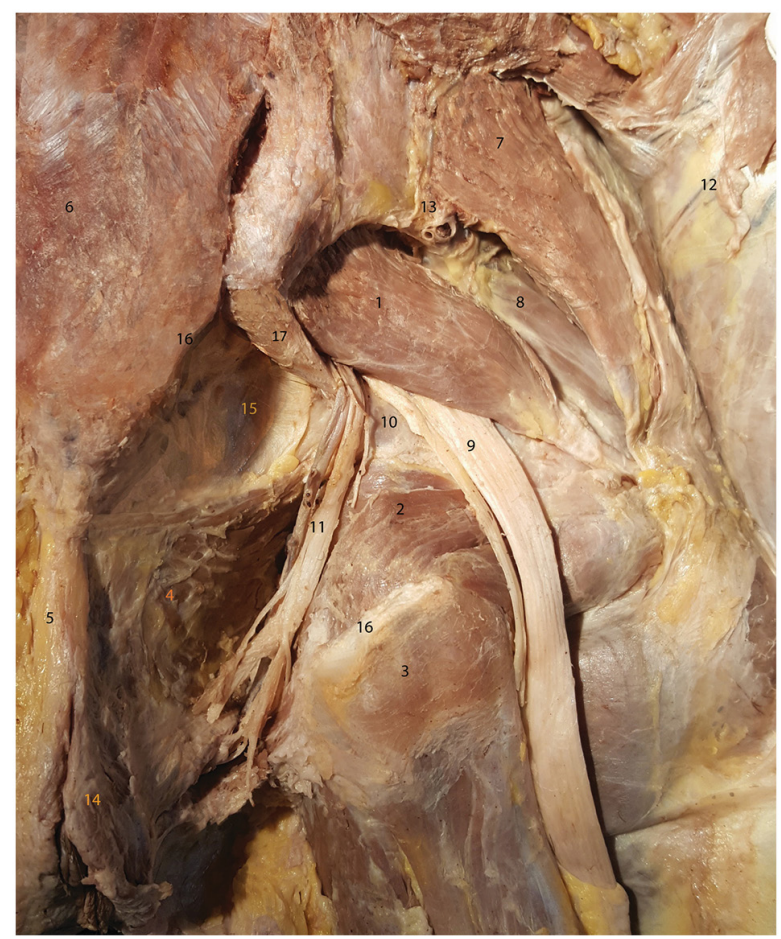

Figure 2. Dissection of the deep gluteal region (posterior view). 1, Piriformis muscle; 2 , internal obturator muscle; 3 , ischial tuberosity (posterior view); 4 , levator ani muscle (superior-medial border of the ischiorectal fossa); 5 , anococcygeal ligament; 6 , sacrum; 7 , gluteus medius muscle; 8 , gluteus minimus muscle; 9 , sciatic nerve; 10 , ischial spine; 11 , internal pudendal neurovascular bundle; 12, gluteus maximus muscle; 13, superior gluteal artery and vein; 14 , external anal sphincter; 15 , pelvic subperitoneal tissue visible after removing the coccygeus muscle; 16 , sacrotuberous attachment points (cut); 17, sacrospinous ligament.

considered as the most significant for the pelvic, extrapelvic and perineal course of the internal pudendal neurovascular bundle were selected (Figs. 1 and 2).

As regards the general anatomical layout, the left half of the pelvic floor is clearly visible. Due to its central location, the sacrospinous ligament and the coccygeus muscle separate the lateral pelvic wall into three zones: i) The posterior-superior zone corresponds to the greater sciatic notch and its content. In a deep plane, the piriformis muscle attaches to the sacrum, with its origins slightly separated by the roots of the sciatic nerve. The anterior divisions of the S2, S3 and S4 spinal nerves give rise to the pudendal nerve. The dissection image clearly shows that these roots can be compressed during the contraction of the piriformis muscle. This nerve, together with the internal pudendal artery, are closely associated with the rigid superior border of the sacrospinous ligament, actually passing between the piriformis muscle and the sacrospinous ligament and then between the ligament and the upper border of the sciatic spine. The elements of the internal pudendal neurovascular bundle follow their extrapelvic course surrounding the ischial spine in the deep gluteal region in order to reenter the pelvis through the lesser sciatic notch. ii) The middle zone is dominated by the attachments of the sacrospinous ligament and coccygeus muscle onto the ischial spine, forming a rigid osteo-fibro-muscular structure. iii) The anterior-inferior zone contains the contents of the lesser ischial notch. In order to reach the internal pudendal neurovascular bundle, the levator 


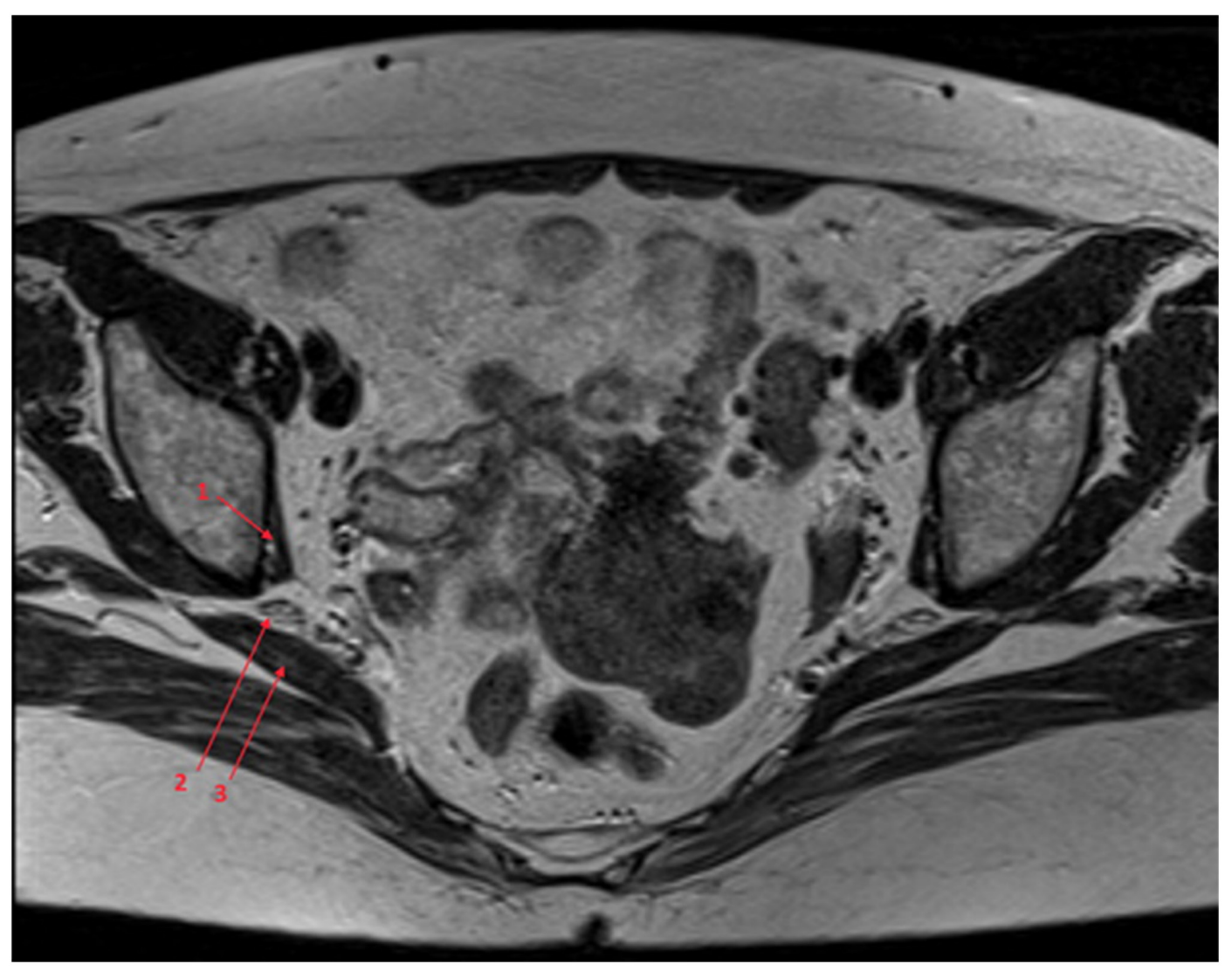

Figure 3. Transverse MRI T2-weighted image at the level of the piriformis muscle. 1, Internal obturator muscle; 2, sciatic nerve; 3, piriformis muscle.

ani muscle was reflected medially, thus obtaining favorable access towards the ischiorectal fossa. On the lateral wall of this fossa, the pudendal canal (Alcock canal) was opened, and the fibrous remnants of the fascia were removed in order to emphasize the neurovascular content of the canal. In close association with the osseous plane, the fascicles of the internal obturator muscle unite in a common body that passes below the inferior border of the ischial spine in its course towards the femoral attachment.

The dissection shown in Fig. 2 was realized in the gluteal region, following the lateral reflection of the gluteus maximus muscle (no. 12).

The image is centered on the emerging sciatic nerve (no. 9) under the inferior border of the piriformis muscle (no. 1), in the infrapiriform space. In the medial end of this space, the internal pudendal neurovascular bundle (no. 11) exits the pelvis, surrounds the ischial spine and reenters the pelvis along the lateral wall of the ischiorectal fossa, in the Alcock canal, which was removed on dissection.

It was observed that the internal obturator and gemellus superior muscles surrounding the lesser sciatic notch run towards its femoral attachment.

The image lacks the sacrotuberous ligament superficially and the coccygeus muscle in the deep plane, as the latter was removed in order to emphasize the connective and adipose tissue within the pelvic subperitoneal space (no. 15).

In the ischiorectal fossa, the inferior rectal branches of the internal pudendal neurovascular bundle lying lateral to the anococcygeal ligament (no. 5) and the external anal sphincter (no. 14) may be clearly seen.
The internal pudendal neurovascular bundle travels through a critical zone between the piriformis muscle and the sacrospinous ligament, in contact with the superior margin of the ischial spine, where compression may occur. When this bundle reenters the pelvis, it may traverse a compression zone between the internal obturator muscle, the gemellus superior muscle and the inferior border of the ischial spine.

The sciatic nerve may become compressed between the inferior margin of the piriformis muscle and the superior margin of the ischial spine.

Observations on imaging examination. A transverse cross-section on MRI at the level of the infrapiriform space is shown in Fig. 3. At this level, the sciatic nerve travels through the anatomical space limited inferiorly by the internal obturator muscle and superiorly by the piriformis muscle.

On the MRI scan shown in Fig. 4, the internal pudendal bundle can be observed reentering the pelvis below the sciatic tuberosity, attaching onto the medial surface of the internal obturator muscle, on the lateral wall of the ischiorectal fossa.

\section{Discussion}

Along the course of the sciatic nerve and the internal pudendal bundle, there are several anatomical associations that may become involved in the compression of the nerve structures, generating the symptoms specific to the piriformis syndrome. The aim of the present study was to establish clear osseous and muscular landmarks for the MRI evaluation of such associations. 


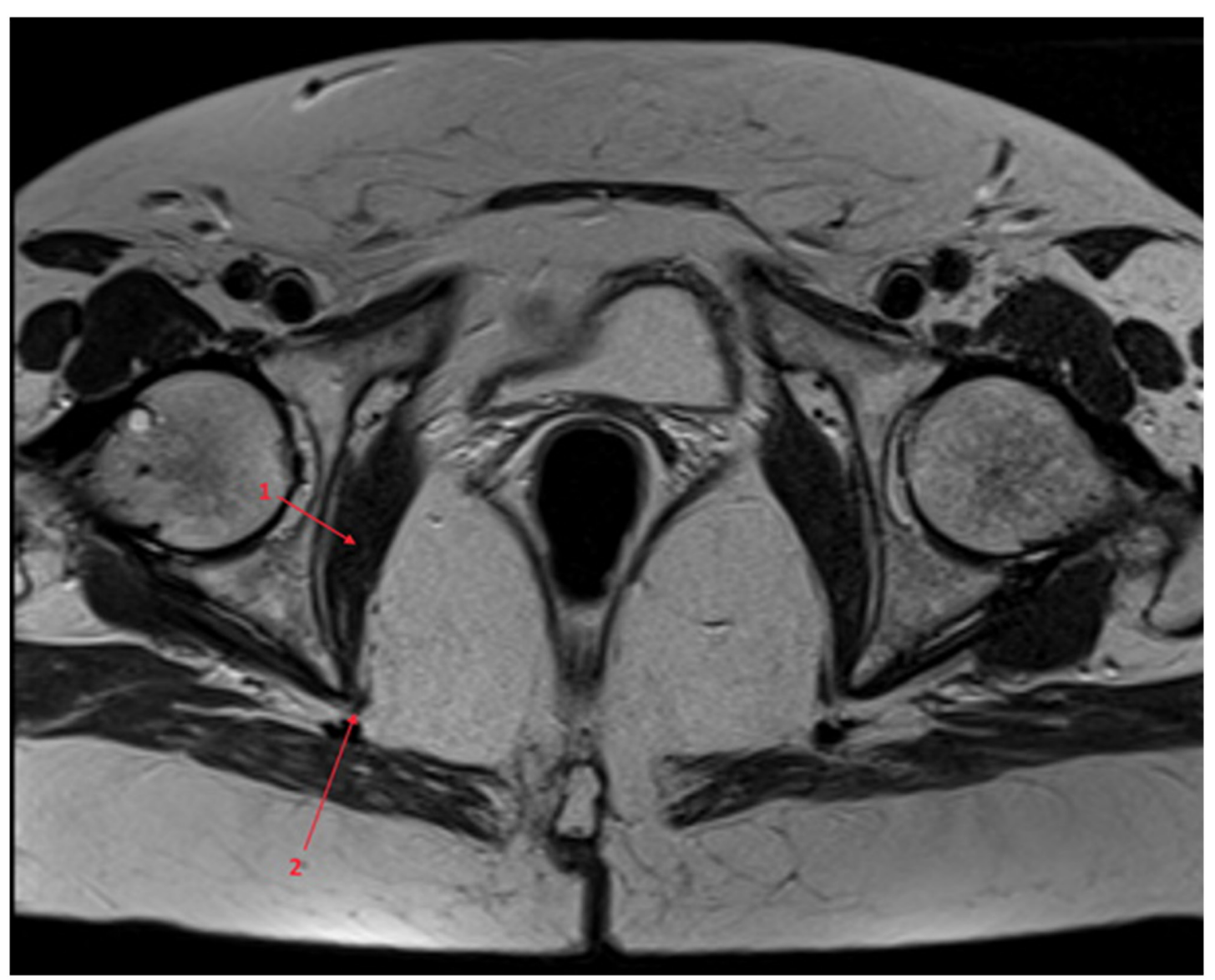

Figure 4. Transverse MRI T2-weighted image at the level of the internal obturator muscle. 1, Internal obturator muscle; 2, internal pudendal neurovascular bundle.

Several aspects are important for the imaging evaluation of the nerve tracts, such as identifying the piriformis muscle in the greater sciatic notch, identifying the internal obturator muscle in its course below the ischial spine, evaluation of the upper and lower borders of the ischial spine, and sequential evaluation of the course of the neurovascular bundles.

The difficulty of the evaluation using MRI lies with the oblique orientation of the sacrum and the piriformis muscle, the curving tendency of the internal obturator muscle and the combined intra- and extrapelvic course of the internal pudendal bundle.

The sciatic nerve can be compressed along its course in the following locations. Its roots can be compressed intrapelvically by the origins of the piriformis muscle; its trunk can be compressed in the infrapiriform space between the inferior border of the piriformis muscle and the upper border of the ischial spine.

The internal pudendal nerve can be compressed along its course in several locations: In its intrapelvic course, it may be compressed in the anterior-inferior part of the piriformis muscle and in the superior border of the sacrospinous ligament. In this part, there are also rare cases of pudendal nerve syndrome entrapment associated with ganglion cysts (19).

Between the piriformis muscle and the border of the greater sciatic notch, there is a space that contains the sciatic nerve (posterior), the internal pudendal and superior gluteal arteries (middle) and the internal pudendal nerve, in direct contact with the upper border of the ischial spine (anterior). At this point, the pudendal nerve is not clearly distinguishable on MRI scans. This is the compression region associated with the definition of the piriformis syndrome.

In its extrapelvic course, the internal pudendal nerve runs in the medial part of the infrapiriform space. In the infrapiriform space, the bundle runs the following course: Upon entering this space, the internal pudendal bundle is actually located between the inferior border of the piriformis muscle and the tip of the ischial spine. Next, the bundle surrounds the lateral aspect of the sciatic spine (Fig. 2) and then reenters the pelvis through the lesser sciatic notch, in contact with the inferior border of the ischial spine passing between the spine, the superior gemellus and internal obturator muscles.

In its intraperineal course, the bundle travels along the internal aspect of the internal obturator muscle, inside the Alcock canal. In this part, the nerve is unlikely to be compressed by extrinsic structures.

The classical piriformis syndrome consists of the compression of the sciatic and/or internal pudendal nerves by the piriformis muscle. It is suggested that the compression of the internal pudendal bundle outside the piriformis muscle may lead to a 'piriformis syndrome-like' symptomatology. We herein explained the anatomical basis for the internal pudendal nerve syndrome, showing the compression zones that may lead to symptoms of perineal pain.

Establishing clear anatomical landmarks and relations in the intra- and extrapelvic course of the sciatic and internal 
pudendal nerves may prove valuable for the evaluation of the course of these nerves through MRI.

\section{Acknowledgements}

Not applicable.

\section{Funding}

No funding was received.

\section{Availability of data and materials}

The data and images used and/or analyzed during the current study are available from the corresponding author on reasonable request.

\section{Authors' contributions}

OCG, ME, ADT, RT, IAV AEN, DD, DI, DG, AM and FMF designed the study, performed a literature search and selected the included studies, and wrote the manuscript. OCG, ME, ADT, RT, IAV AEN, DD, DI, DG, AM and FMF critically revised the manuscript for important intellectual content. $\mathrm{ME}$, FMF and ADT confirm the authenticity of the raw data. All the authors have read and approved the final manuscript.

\section{Ethics approval and consent to participate}

The cadavers used in the present study were provided by the Department of Morphological Sciences, Discipline of Anatomy of the University of Medicine and Pharmacy (Bucharest, Romania). The Research Ethics Committee of the University of Medicine and Pharmacy 'Carol Davila' approved the use of human cadavers for the purposes of this study (approval no. 210763/06.10.2021). The Ethics Committee of the 'Victor Babeş' Diagnosis and Treatment Center approved the use of pelvic MRI examination for the purpose of this study (approval no. 1647/03.06.2020).

\section{Patient consent for publication}

Not applicable.

\section{Competing interests}

The authors declare that they have no competing interests.

\section{References}

1. Anbumani TL, Thamarai SA and Anthony AS: Sciatic nerve and its variations: An anatomical study. Int J Anat Res 3: 1121-1127, 2015.

2. Ripani M, Continenza MA, Cacchio A, Barile A, Parisi A and De Paulis F: The ischiatic region: Normal and MRI anatomy. J Sports Med Phys Fitness 46: 468-475, 2006.

3. Chang C, Jeno SH and Varacallo M: Anatomy, bony pelvis and lower limb, piriformis muscle. In: StatPearls. Treasure Island (FL): StatPearls Publishing, 2021.

4. Hopayian K, Song F and Sambandan RRS: The clinical features of the piriformis syndrome: A systematic review. Eur Spine J 19: 2095-2109, 2010.

5. Carro LP, Hernando MF, Cerezal L, Navarro IS, Fernandez AF and Castillo AO: Deep gluteal space problems: Piriformis syndrome, ischiofemoral impingement and sciatic nerve release. Muscles Ligaments Tendons J 6: 384-396, 2016.

6. Beaton LE and Anson BJ: The relation of the sciatic nerve and its subdivisions to the piriformis muscle. Anat Rec 70: 1-5, 1937.

7. Pećina M: Contribution to the etiological explanation of the piriformis syndrome. Acta Anat (Basel) 105: 181-187, 1979.

8. Vij N, Kiernan H, Bisht R, Singleton I, Cornet EM, Kaye AD, Imani F, Varassi G, Pourbahiri M, Viswanath O and Urits I: Surgical and non-surgical treatment options for piriformis syndrome: A literature review. Anesth Pain Med 11: e112825, 2021.

9. Yeoman W: The relation of arthritis of the sacro-iliac joint to sciatica, with an analysis of 100 cases. Lancet 212: 1119-1123, 1928.

10. Freiburg AH and Vinke TA: Sciatica and the sacroiliac joint. J Bone Joint Surg 16: 126-36, 1934.

11. Beaton LE and Anson BJ: The sciatic nerve and the piriformis muscle. Their interrelation a possible cause of coccygodynia. J Bone Joint Surg 20: 686-688, 1938.

12. Robinson D: Piriformis syndrome in relation to sciatic pain. Am J Surg 73: 356-358, 1947.

13. Hopayian K and Danielyan A: Four symptoms define the piriformis syndrome: An updated systematic review of its clinical features. Eur J Orthop Surg Traumatol 28: 155-164, 2018.

14. Papadopoulos EC and Khan SN: Piriformis syndrome and low back pain: A new classification and review of the literature. Orthop Clin North Am 35: 65-71, 2004.

15. Hopayian K and Heathcote J: Deep gluteal syndrome: An overlooked cause of sciatica. Br J Gen Pract 69: 485-486, 2019.

16. Martin HD, Kivlan BR, Palmer IJ and Martin RL: Diagnostic accuracy of clinical tests for sciatic nerve entrapment in the gluteal region. Knee Surg Sports Traumatol Arthrosc 22: 882-888, 2014.

17. Martin HD, Shears SA, Johnson JC, Smathers AM and Palmer IJ: The endoscopic treatment of sciatic nerve entrapment/deep gluteal syndrome. Arthroscopy 27: 172-181, 2011.

18. Park JW, Lee YK, Lee YJ, Shin S, Kang Y and Koo KH: Deep gluteal syndrome as a cause of posterior hip pain and sciatica-like pain. Bone Joint J 102: 556-557, 2020.

19. Kim YJ and Kim DH: Pudendal nerve entrapment syndrome caused by ganglion cysts along the pudendal nerve. Yeungnam Univ J Med 38: 148-151, 2021. 